\title{
Measuring Topological Chaos
}

\author{
Jean-Luc Thiffeaul $*$ \\ Department of Mathematics, Imperial College London, SW7 2AZ, United Kingdom
}

\begin{abstract}
The orbits of fluid particles in two dimensions effectively act as topological obstacles to material lines. A spacetime plot of the orbits of such particles can be regarded as a braid whose properties reflect the underlying dynamics. For a chaotic flow, the braid generated by the motion of three or more fluid particles is computed. A "braiding exponent" is then defined to characterize the complexity of the braid. This exponent is proportional to the usual Lyapunov exponent of the flow, associated with separation of nearby trajectories. Measuring chaos in this manner has several advantages, especially from the experimental viewpoint, since neither nearby trajectories nor derivatives of the velocity field are needed.
\end{abstract}

Boyland et al. [1] showed that strong chaos can be induced through topological obstacles moving in a twodimensional fluid. Their system consisted of three rods whose repeated interchange led to complicated braiding of material lines. Chaos ensued as a consequence of the topology: Thurston-Nielsen theory [2, 3] guarantees the existence of a region of the flow which has pseudo-Anosov dynamics. In practical terms this means this region has extremely strong chaotic properties - almost every point exhibits exponential stretching-leading to good mixing [4]. Thurston-Nielsen theory does not say how large the pseudo-Anosov region is (it could even have zero measure), but in physical and numerical experiments it has usually been found to be sizeable and localized near the rods (though see [5] for an example where the region is too small to be important for mixing).

Physically speaking, material lines "snag" on the rods, and if the rods have a complicated braiding motion then the length of material lines must grow exponentially.

More recently, Boyland et al. [6] applied the theory where vortices serve as topological obstacles. Several vortices orbited each other and the authors classified the braiding properties of the different configurations. Vikhansky 7] pushed this a step further: he studied the properties of freely-moving rods (which he called discs) in a two-dimensional cavity flow. In this case the braiding arises from the chaotic motion of the rods. By mapping each element of the braid group to its matrix representation, a Lyapunov exponent can be found which characterizes the vigor of topological chaos.

In this letter we apply topological techniques as diagnostic tools to quantify chaos in general two-dimensional dynamical systems. In the absence of diffusion, in a two-dimensional bounded domain any fluid particle (Lagrangian tracer) is a topological obstacle to material lines. That is, if we choose a material line connected to the boundary and a reference fluid particle (not on the material line), both moving with the fluid, then the material line must bend around the fluid particle as they move. Deterministic motion forbids the crossing of the line and the fluid particle, since at the moment of cross- ing the fluid particle would have to belong to the material line, and it must thus have belonged to it for its entire history.

Since they are topological obstacles, any $n$ fluid particles can be seen as candidates for topological chaos: the motion of the particles can exhibit complex braiding. Thus, we can look for the presence of topological chaos by following $n$-tuplets of fluid particles and recording their braiding history - an ordered sequence of braid group generators. We then use an appropriate matrix representation of the braid group to express this sequence as a matrix product.

From random matrix theory, we can expect the resulting sequence to have eigenvalues that grow or decay exponentially with well-defined Lyapunov exponents, which we call braiding exponents. If one of these exponents is positive, then we say that the system exhibits topological chaos. We will show by an example that the magnitude of this largest exponent is proportional to the usual Lyapunov exponent of the flow, defined in terms of separation of nearby trajectories. The work presented here is meant to be a step in the development of what was aptly described as "topological kinematics" by Boyland et al. [1].

An obvious advantage of this method over measuring the chaotic properties of a system by computing the usual Lyapunov exponents is that there is no need for closelyspaced trajectories. If an experiment yields data for a large number of fluid trajectories, then all possible $n$ tuplets can be used to compute the topological chaos properties (as long as they belong to the same chaotic region). Neither the velocity field nor its spatial derivatives are needed; the only issue is whether the time series is of sufficient length. (For convenience we use the language of two-dimensional fluids, but the method presented here can be applied to any two-dimensional deterministic flow.)

Historically, the focus was on periodic particle orbits before moving rods were considered [3]. Here the new ingredient is that the orbits are chaotic and determined explicitly by numerical methods. The association of arbi- 


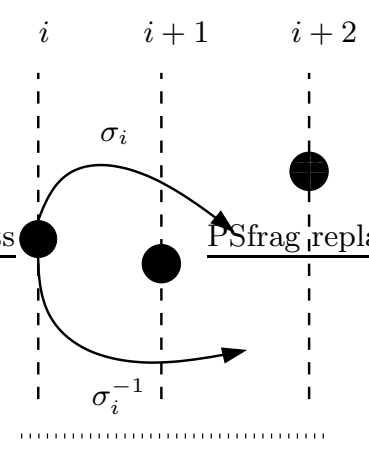

(a)

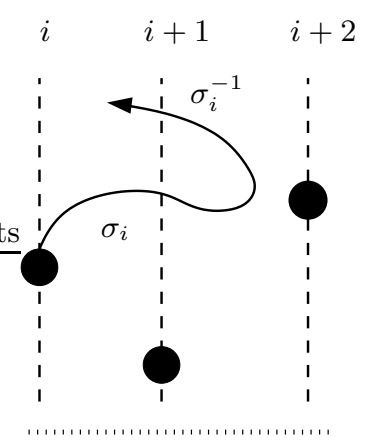

(b)
FIG. 1: Detecting crossings: (a) Two possible particle paths that are associated with different braid group generators; (b) Two crossings that yield no net braiding. The reference line used to detect crossings is shown dotted, and the perpendicular lines used to determine the braid generator are shown dashed.

trary particle orbits with braids group elements was first proposed by Gambaudo and Pécou [8].

We now describe the method, which we mean to keep as simple as possible for easy implementation. This is essentially the same technique as used by Boyland et al. [6] for the braiding of vortices and Vikhansky [7] for freelymoving discs. Our goal is to map the motion of $n$ particles onto elements of the braid group.

First project the position of the particles onto any fixed reference line (which we choose to be the horizontal axis), and label the particles by $i=1,2, \ldots, n$ in increasing order of their projection [13]. A crossing occurs whenever two particles interchange position on the reference line. A crossing can occur as an "over" or "under" braid, which for us means a clockwise or counterclockwise interchange. We define the braid $\sigma_{i}$ as the clockwise interchange of the $i$ th and $(i+1)$ th particles, and $\sigma_{i}^{-1}$ as their counterclockwise interchange, for $i=1, \ldots, n-1$. These elementary braids are the generators of the Artin $n$-braid group [9].

Assuming a crossing has occurred between the $i$ th and $(i+1)$ th particles, we need to determine if the corresponding braid generator is $\sigma_{i}$ or $\sigma_{i}^{-1}$. Look at the projection of the $i$ th and $(i+1)$ th particles in the direction perpendicular to the reference line (the vertical axis in our case). If the $i$ th particle is above the $(i+1)$ th at the time of crossing, then the interchange involves the group generator $\sigma_{i}$ (we define "above" as having a greater value of projection along the perpendicular direction). Conversely, if the $i$ th particle is below the $(i+1)$ th at the time of crossing, then the interchange involves the group generator $\sigma_{i}^{-1}$. Figure 1(a) depicts these two situations.

The method just described might seem to detect spurious braids if two well-separated particles just happen to interchange position several times in a row on the reference line, as shown in Figure 1(b) However, this would imply a sequence of $\sigma_{i}$ and $\sigma_{i}^{-1}$ braids, since which particle is the $i$ th one changes at each crossing. When composed together these crossings produce no net braiding at all.

We now select a matrix representation for the generators of the braid group [1, 7]. These matrices are given by the Burau representation [10, 11] of the $n$-braid group, which consists of $(n-1) \times(n-1)$ matrices defined by

$$
\left[\sigma_{i}\right]_{k \ell}=\delta_{k \ell}+\delta_{k, i-1} \delta_{\ell i}-\delta_{k, i+1} \delta_{\ell i},
$$

with inverses

$$
\left[\sigma_{i}^{-1}\right]_{k \ell}=\delta_{k \ell}-\delta_{k, i-1} \delta_{\ell i}+\delta_{k, i+1} \delta_{\ell i},
$$

where $i, k, \ell=1, \ldots, n-1$ and we set $\delta_{k, 0}$ and $\delta_{k, n}$ to zero. (For simplicity, we do not distinguish between the elements of the braid group and their matrix representation.) The determinant of each of these matrices is unity, and they satisfy the "physical braid" conditions [9] : $\sigma_{i} \sigma_{j}=\sigma_{j} \sigma_{i}$ for $|i-j| \geq 2$, and $\sigma_{i} \sigma_{i+1} \sigma_{i}=\sigma_{i+1} \sigma_{i} \sigma_{i+1}$. The matrices (11)-(2) can be understood as arising from the lengthening of line segments tied to the particles as the particles braid around each other [1, 7].

As we detect crossings, we compute the running product $\Sigma^{(N)}$ of all the braid group elements,

$$
\Sigma^{(N)}=\sigma^{(N)} \cdots \sigma^{(2)} \sigma^{(1)}
$$

where $\sigma^{(\mu)} \in\left\{\sigma_{i}, \sigma_{i}^{-1} \mid i=1,2, \ldots, n-1\right\}$ and $N(t)$ is the number of crossings detected after a time $t$. Now $\Sigma^{(N)}$ is the product of a sequence of (possibly random) matrices. We define the braiding factor to be the largest eigenvalue of $\Sigma^{(N)}$. According to Oseledec's multiplicative theorem [12], we can express the time-asymptotic exponential growth rate of the braiding factor by a (nonnegative) Lyapunov exponent, which we call the braiding exponent, defined by

$$
\text { braiding exponent }=\lim _{t \rightarrow \infty} \frac{1}{t} \log \mid \text { braiding factor } \mid .
$$

The braiding exponent is a function of the number $n$ of braiding particles. If the exponent is positive, then we say that the sequence of braids exhibits topological chaos. Note that the braiding exponent has units of inverse time, so that if the frequency of crossings decreases then the exponent also decreases.

We illustrate the method using the blinking vortex flow (eggbeater flow) of Aref [4]. The flow consists of two spatially-fixed vortices with opposite circulation that act alternately in time. It has the advantage of being confined to a bounded circular domain and of exhibiting chaos for a large enough value of the circulation $\Gamma$.

Figure 2(a) shows the braiding factor for three particles $(n=3)$ as a function of time for $\Gamma=0.5$. For the 


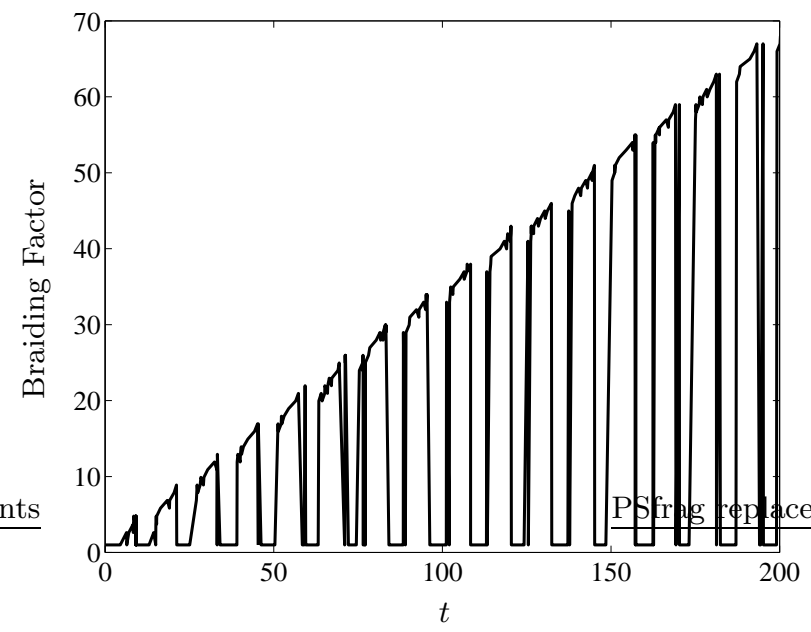

(a)

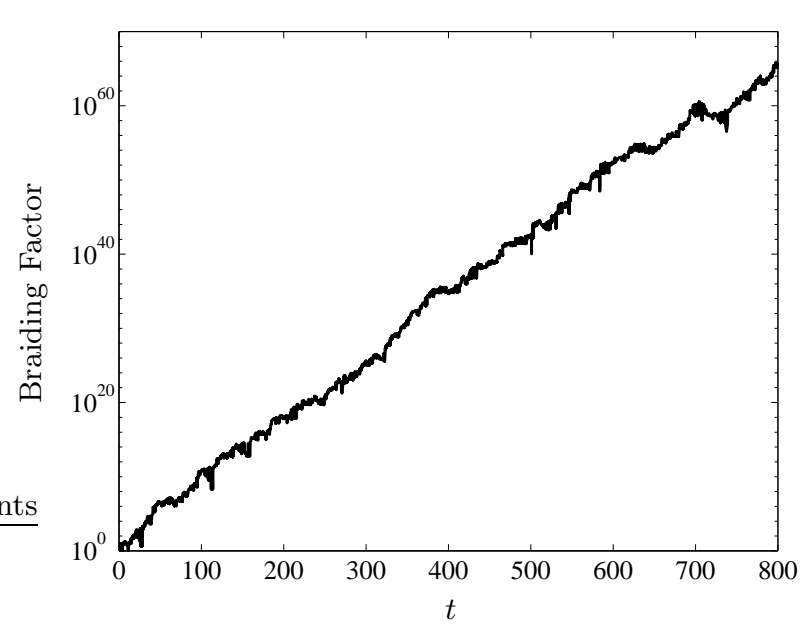

(b)

FIG. 2: Braiding factor (largest eigenvalue of $\Sigma^{(N)}$ ) as a function of time for a triplet of particles (a) without topological chaos $(\Gamma=0.5)$; (b) with topological chaos $(\Gamma=13)$. Note that in (b) the vertical axis is logarithmic.

initial condition chosen, only one particle is undergoing chaotic motion; the other two move in periodic orbits with incommensurate frequencies. Two features are evident in Figure 2(a) the braiding factor grows more or less linearly, and it regularly returns to unity. Because of the linear growth, the braiding exponent for this triplet of trajectories is zero.

Figure 2(b) shows the same system (with the same initial condition for the triplet of trajectories) but for $\Gamma=$ 13 , making the system chaotic almost everywhere. In this case, the braiding factor grows exponentially, and it never returns to unity (except at the very beginning). This is the signature of topological chaos: the braiding exponent for the triplet is about 0.2. For large enough time, the exponent is the same for any triplet initially within the same chaotic region.

Of course, the braiding exponent is most useful if it correlates well with the Lyapunov exponent based on the exponential separation of trajectories. The braiding exponent is a lower bound on the rate of stretching of material lines [1, 7] (the topological entropy), which in turn is correlated with the Lyapunov exponent. Figure 3 shows the two types of exponents (braiding and Lyapunov) plotted against each other for different values of the circulation in the blinking vortex flow. The relationship between the two is almost linear: both the Lyapunov and braiding exponents increase monotonically with circulation. This is consistent with the exponents measuring the same underlying "degree of chaos". There is a similar correlation between the braiding exponent and the rate of stretching of material lines.

Figure 4 shows the behavior of the braiding exponent as a function of the number $n$ of braiding particles.

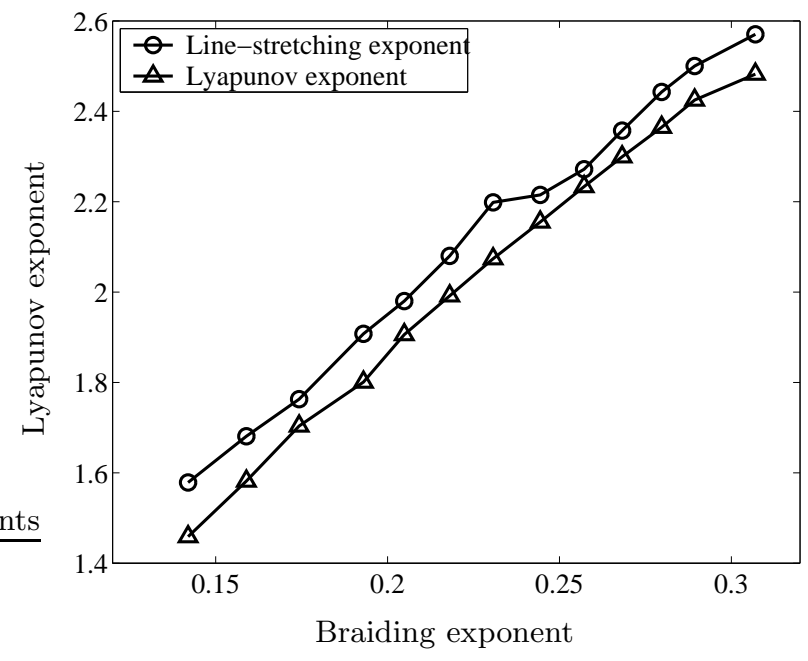

FIG. 3: Line-stretching and Lyapunov exponents plotted versus the braiding exponent for three particles $(n=3)$ and circulation $\Gamma$ varying from 8 to 20 . All the exponents increase monotonically with circulation.

For $n=1$ the braiding exponent is undefined, since one particle cannot braid around itself. For $n=2$ the exponent is always zero (our representation of the 2-braid group contains only the identity). For $n \geq 3$ the exponent evolves along different curves depending on whether $n$ is odd or even; these converge together for large $n$. Since the braiding exponent is a lower bound on the topological entropy of the flow, the curve should saturate for large $n$ : simulations were performed all the way to $n=100$ (not shown) with slow growth of the braiding exponent but no sign of saturation. One reason for the growth of the 


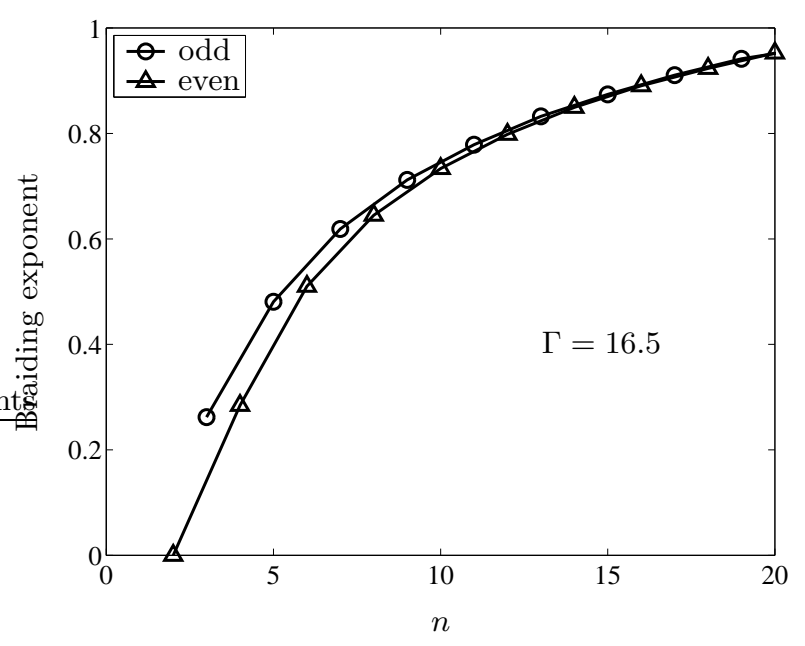

FIG. 4: Braiding exponent as a function of the number of braiding particle orbits, for an odd and even number of particles.

braiding exponent with $n$ is simply that more braiding particles give more crossings for a given length of time. It may thus be advantageous to use many particles when the length of the time series is limited, as long as the particles exhibit sufficiently nontrivial braiding (not simply crossing over each other as in Fig. 1(b)]. Note that the $n$ particle braiding exponent exhibits a linear relationship with the Lyapunov exponent, just as it did for $n=3$ (Fig. 3).

The diagnostic approach presented here sheds some light on Vikhansky's results [7]: he observed an increase in the chaotic stretching properties of his cavity flow of about $30 \%$ when freely-moving rods were present. Compared to the topological effect observed by Boyland et al. [1], this is a modest increase. This is because the rods in Vikhansky's case are no more topological obstacles than any fluid particle. Their presence modifies the flow and so increases its chaotic properties, but this is not a topological effect. In other words, in Vikhan- sky's case the chaos is already present even without rods, whereas in Boyland et al. the rods cause the chaos.

The measure of chaos presented here is attractive because of its emphasis of global aspects of the flow. Lyapunov exponents are difficult to measure because we try to infer from them a global quantity (the Lyapunov exponent associated with a given region of the flow) from local measurements (the rate of separation of nearby trajectories). These local measurements are difficult for an experimentalist to make, so we suggest focusing on the global braiding of particles instead.

The author thanks Francesco Paparella, Joseph Lacasce, Edward Spiegel, Philip Boyland, Matthew Finn, and Andrew Gilbert for helpful discussions.

* Electronic address: jeanluc@imperial.ac.uk

[1] P. L. Boyland, H. Aref, and M. A. Stremler, J. Fluid Mech. 403, 277 (2000).

[2] W. Thurston, Bull. Am. Math. Soc. 19, 417 (1988).

[3] P. L. Boyland, Topology and Its Applications 58, 223 (1994).

[4] H. Aref, J. Fluid Mech. 143, 1 (1984).

[5] M. D. Finn, S. M. Cox, and H. M. Byrne, Phys. Fluids 15, L77 (2003).

[6] P. L. Boyland, M. A. Stremler, and H. Aref, Physica D 175, 69 (2003).

[7] A. Vikhansky, Phys. Fluids 15, 1830 (2003).

[8] J.-M. Gambaudo and E. E. Pécou, Ergod. Th. Dynam. Sys. 19, 627 (1999).

[9] K. Murasugi, Knot theory and its applications (Birkhäuser,, Boston, 1996).

[10] W. Burau, Abh. Math. Sem. Hanischen Univ. 11, 171 (1936).

[11] V. F. R. Jones, Ann. Math. 126, 335 (1987).

[12] V. I. Oseledec, Trans. Moscow Math. Soc. 19, 197 (1968).

[13] Unlike 6], we are not dealing with periodic orbits. so a change of reference line does not simply lead to conjugation by another braid word. However, the braid word is changed by only a finite number of braid generators, which does not influence the braiding exponent. 